\title{
MIDIENDO LA CALIDAD: UNA OPINIÓN PARTICULAR
}

\section{MEASURING QUALITY: A PARTICULAR VIEW}

\section{AUTORES}

Horacio Rico Lenza: Catedrático de Medicina. Universidad de Alcalá horacio.rico@arrakis.es

\section{CURRÍCULUM VITAE}

Catedrático de Clínica Médica de la Universidad de Alcalá.

\section{RESUMEN}

Entre el Ministerio y la Universidad, así como entre las distintas Universidades y sus miembros funcionarios docentes (Profesores Numerarios de los Cuerpos Docentes Universitarios), hay una continua querella relativa a la "evaluación" de la calidad investigadora, a la que nos referiremos en este caso; buen ejemplo de ello fueron las tremendas y airadas quejas y enfados derivados de aquellos a los que, inicialmente, se les negó el sexenio de Investigación.

\section{PALABRAS CLAVE}

Calidad - Opinión - Universidad 


\begin{abstract}
Between the Ministry and the University as well as between different universities and teaching staff members (tenured professors of the university faculty), there is an ongoing lawsuit concerning the "evaluation" of research quality, which we refer in this case; good example of this was the terrible and angry complaints and anger arising from those who initially denied the presidency of Research.
\end{abstract}

\title{
KEY WORDS
}

Quality - Review - University

Entre el Ministerio y la Universidad, así como entre las distintas Universidades y sus miembros funcionarios docentes (Profesores Numerarios de los Cuerpos Docentes Universitarios), hay una continua querella relativa a la "evaluación" de la calidad investigadora, a la que nos referiremos en este caso; buen ejemplo de ello fueron las tremendas y airadas quejas y enfados derivados de aquellos a los que, inicialmente, se les negó el sexenio de Investigación.

No conocemos muy a fondo el conjunto general de los criterios de la Comisión Nacional Evaluadora, pero nos consta que uno de los primeros es el actualmente generalizado "factor de impacto" o puntuación asignada a una investigación "publicada" en una revista que tiene un determinado "factor de impacto", mayor o menor según su calidad científica y el prestigio internacional de la misma. Éste, con argumentos controvertidos, es negativamente criticado por aquellos que no tienen un adecuado "factor de impacto" en sus curriculum, mientras que la comunidad científica mundial lo acepta con muy buen grado, dado que día a día se consolida con 
más eficiencia y seguridad como una medida muy adecuada, transparente y totalmente objetiva, para evaluar la calidad de una determinada investigación y consecuentemente de los que la llevaron a efecto.

En este sentido, es evidente y lógico el que existan diferencias entre las disciplinas de ciencias experimentales y las de letras o humanidades. Pensamos que la solución, para equiparar estas diferencias en el factor de impacto, es sencilla, práctica y fácilmente aplicable. Dependiendo de la disciplina, a la revista con máxima puntuación se le asigna el 100\% del valor y el valor aplicable es este porcentaje, a continuación al resto de las revistas, según su factor de impacto, se les aplica dicho porcentaje; un ejemplo lo vemos en la tabla 1, donde se hace dicha suma (total) dependiendo del tipo de revista y de que ésta la consideremos en el índice general, por ciencias (siempre el máximo corresponde a las revistas de ciencias, por lo que sería superponible al índice general), por letras $y$, dentro de las ciencias, lo que también se puede hacer para las de letras, dependiendo de la especialidad. Así, si la revista de máxima puntuación es de 56 tiene el 100\%, si lo es la segunda con un 50,4 tiene un 90\% y una intermedia de 3,25 tendrá un 5,8\%, la suma de los porcentajes es lo que se debe aplicar y de esta manera, como podemos ver en la tabla 1, independientemente del mayor o menor factor de impacto de la revista, al aplicar el porcentaje, el factor de impacto acumulado se normaliza.

Puede haber casos excepcionales, como por ejemplo, en Medicina, un Cirujano que publique en una revista de Medicina Interna, usualmente de mayor factor de impacto que las quirúrgicas. Creemos que es muy sencillo, se le aplica, en la tabla correspondiente a su especialidad, el valor de dicha revista con lógica satisfacción del autor o autores, lo contrario, obviamente, ocurriría si publica en una revista, de otra especialidad, con menor factor de impacto. Aunque no muy adecuada esta "normalización", su sencillez y generalización minimizan las diferencias que se puedan derivar de esta aplicación.

No queremos cuestionarnos el lugar que un autor determinado ocupa, entre los firmantes, en el trabajo de investigación publicado. Al menos en Medicina, el autor 
principal o responsable, suele ir en el primer o último puesto, pero cada día es norma general mayor, por las revistas científicas, de que el trabajo vaya acompañado de una carta con el visto bueno de todos los autores y en la que se exige el que todos hayan contribuido de manera apreciable en el desarrollo de la investigación; por ello, si todos han tenido una contribución apreciable, quiere decirse que su labor fue imprescindible para llevar a efecto la publicación y, consecuentemente, todos tienen el mismo mérito y puntuación, dado que a falta de la contribución de uno de ellos, el trabajo quizá no se hubiese podido realizar y/o finalizar.

Los trabajos publicados en libros, a nuestro modo de ver, no deben ser evaluados, forman parte más del capítulo de Docencia que del de Investigación y la evaluación de los tramos docentes corresponde a las Universidades, previo informe preceptivo de los Departamentos. Son escasas las ocasiones en que una investigación da lugar a la publicación de un libro en un sentido estricto, de ocurrir, formaría parte de un capítulo extraordinario en la evaluación, que obligaría a establecer unos criterios adecuados, que bien pudieran ser el de aplicar al mismo el baremo según el factor de impacto en atención a los trabajos de investigación, publicados, e incluidos en el libro.

No consideramos oportuno y lógico el puntuar o baremar las aportaciones a Congresos o Reuniones, todos sabemos que si se paga la inscripción, en la mayoría de ellos, como es usual en Medicina, aceptan todo lo que se envía; por ello consideramos que no deben ser evaluados; por otro lado si son buenas aportaciones suelen publicarse y, en este sentido, ya son valorados. Si la aportación es mala y no llega a ser publicada, es obvio que no debe ser considerada como investigación puntuable. Lo mismo, a nuestro modo de ver, es aplicable a las Tesis Doctorales, sólo deben valorarse, con criterios de Investigación ya que es lo que deben ser (investigaciones de elevado rango), cuando demuestren la calidad de la misma a través de las publicaciones al respecto que emanen de su desarrollo y conclusiones. Es obvio que es totalmente discriminativo dar la misma puntuación, como norma, a una Tesis que no llega a publicarse (por desgracia todavía hoy en día la mayoría), 
que a aquella de la que derivan una o más publicaciones científicas nacionales o internacionales al respecto, y que consecuentemente aportan también una mayor difusión cultural y científica, para general conocimiento de la sociedad y comunidad científica.

Otros criterios son también, en lo que a la investigación científica y su valoración se refiere, obligatorios de cuantificar y evaluar y, por desgracia, en la mayor parte de las veces no se hace y de hacerlo no se lleva a efecto, a nuestra manera de ver, de forma adecuada. El nivel de Investigación en un Departamento o en cualquier Centro de Investigación, hoy como hemos visto es objetivamente medible, y debe estar en relación directa con los medios humanos y materiales disponibles, de no ser así hay un desequilibrio que, obviamente, debe analizarse con criterios éticos y objetivos y posteriormente remediar. Para ello una manera sencilla, también objetiva, es que dependiendo de los medios materiales y/o infraestructura y los humanos, y puntuados de forma adecuada, se establezca un mínimo de investigación que se debe exigir y conseguir. De no hacerse esta investigación exigible, debe penalizarse de la manera que se estime más oportuna, pero nunca dejar de hacerlo, dado que el principio básico e ineludible de la Universidad es la Investigación y la Docencia y como universitarios debemos cumplir con aquello que la sociedad nos demanda y para lo que nos paga, mejor o peor, pero que nosotros aceptamos, por lo que consecuentemente estamos obligados y debemos corresponder.

Las concesiones de las ayudas económicas para la investigación, también deben ser consideradas de una manera especial. Hay tipos de ayudas, todos los sabemos, que tienen un porcentaje muy alto del beneficio de la amistad, e incluso de condicionamientos políticos, lo que por desgracia repercute en el bolsillo de todos los ciudadanos y en el deterioro de nuestras Universidades. Por ello y para evitar estos y otros tipos de abusos, al conceder una ayuda económica debe especificarse que de la misma, y dependiendo de su cuantía, se espera el aporte de una determinada investigación de calidad; en ella, obviamente, no sólo influye la ayuda económica, sino también la propia infraestructura humana y material de aquel o aquellos a los 
que se les concedió la ayuda, así la fórmula de: asignación concedida / medios materiales y humanos disponibles $=$ mínimo de investigación de calidad exigible $\mathrm{a}$ conseguir. Ello, aunque la ayuda sea para incorporación de nueva infraestructura o renovación de material obsoleto, de ambos, a corto o medio plazo, es exigible el esperar unos frutos adecuados. De no obtenerlos, nuevamente pensamos que se debe demandar una adecuada explicación, la que, de no ser convincente, hay que penalizar de forma contundente, para acabar con los abusos que siempre se han cometido, y que por desgracia se siguen tolerablemente cometiendo.

La adecuada aplicación y exigencia de estas premisas, es una labor ineludible de toda Universidad; el no tomarlas en consideración y no exigir que se cumplan de forma satisfactoria, no sólo va en perjuicio de la Universidad que no lo hace, sino que genera situaciones totalmente discriminativas e inconstitucionales, por cuanto se beneficia a unos en perjuicio de los demás, lo que obviamente con los criterios de adecuada transparencia debemos evitar, con un único fin, obligado a nuestra condición de universitarios, el que tenemos para contribuir a que nuestra comunidad científica aumente y mejore su calidad día a día.

Es obvio que la opinión aquí vertida pueda chocar con criterios a aplicar en las disciplinas de humanidades, dependientes a su vez de su diversidad y de las tendencias históricas propias, pero consideramos que hoy en día todo es objetivamente medible y cuantificable, y consecuentemente superponible para todo aquello que forme parte de lo que se denomina Ciencia, ya lo sea en el terreno experimental o en el de las humanidades. 
Tabla 1. Supuesto factor de impacto (F. I.) en la clasificación de una "determinada revista científica", ya lo sea en el computo total de las mismas (general y/o ciencias), en el de las de letras y en el de las divididas por especialidades, dependientes, igualmente, de que lo sean de ciencias (experimentales) o letras (humanidades).

\begin{tabular}{|c|c|c|c|c|c|c||}
\hline Clasificación & $\begin{array}{c}\text { F.I. } \\
\text { General }\end{array}$ & $\begin{array}{c}\text { Valor } \\
\%\end{array}$ & F. I. Letras & $\begin{array}{c}\text { Valor } \\
\%\end{array}$ & $\begin{array}{c}\text { F. I. } \\
\text { Especialidad }\end{array}$ & $\begin{array}{c}\text { Valor } \\
\%\end{array}$ \\
\hline \hline $1^{\text {a }}$ & 56,00 & 100,0 & 4,00 & 100,0 & 21,0 & 100,00 \\
\hline \hline $2^{a}$ & 50,40 & 90,0 & 3,60 & 90,0 & 18,9 & 90,00 \\
\hline \hline Intermedia & 3,25 & 5,8 & 0,23 & 5,8 & 1,2 & 5,80 \\
\hline \hline Total & & 195,8 & & 195,8 & & 195,8 \\
\hline \hline
\end{tabular}

